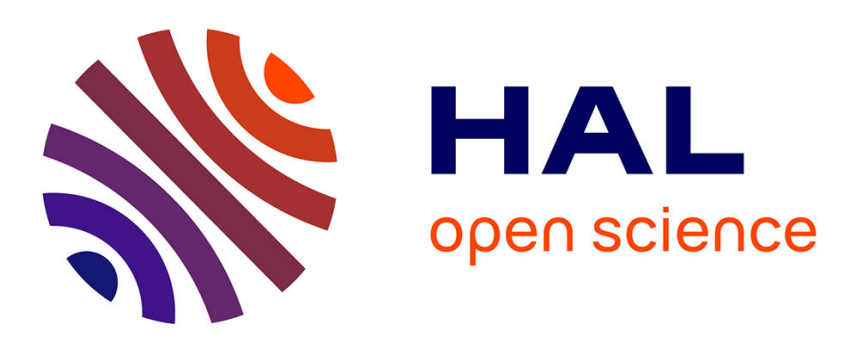

\title{
A perturbation-method-based post-processing for the planewave discretization of Kohn-Sham models
}

Eric Cancès, Geneviève Dusson, Yvon Maday, Benjamin Stamm, Martin Vohralík

\section{- To cite this version:}

Eric Cancès, Geneviève Dusson, Yvon Maday, Benjamin Stamm, Martin Vohralík. A perturbationmethod-based post-processing for the planewave discretization of Kohn-Sham models. Journal of Computational Physics, 2016, 307, pp.446-459. 10.1016/j.jcp.2015.12.012 . hal-01140818v2

\section{HAL Id: hal-01140818 \\ https://hal.sorbonne-universite.fr/hal-01140818v2}

Submitted on 15 Oct 2015

HAL is a multi-disciplinary open access archive for the deposit and dissemination of scientific research documents, whether they are published or not. The documents may come from teaching and research institutions in France or abroad, or from public or private research centers.
L'archive ouverte pluridisciplinaire HAL, est destinée au dépôt et à la diffusion de documents scientifiques de niveau recherche, publiés ou non, émanant des établissements d'enseignement et de recherche français ou étrangers, des laboratoires publics ou privés. 


\title{
A perturbation-method-based post-processing for the planewave discretization of Kohn-Sham models
}

\author{
Eric Cancès ${ }^{\mathrm{a}}$, Geneviève Dusson ${ }^{\mathrm{b}, \mathrm{c}}$, Yvon Maday ${ }^{\mathrm{b}, \mathrm{d}, \mathrm{e}}$, Benjamin Stamm ${ }^{\mathrm{b}}$, Martin Vohralík ${ }^{\mathrm{f}}$ \\ a Université Paris Est, CERMICS, Ecole des Ponts and INRIA, 6858 Av. Pascal, 77455 Marne-la-Vallée, \\ France \\ ${ }^{b}$ Sorbonne Universités, UPMC Univ. Paris 06 and CNRS, UMR 7598, Laboratoire Jacques-Louis Lions, \\ F-75005, Paris, France \\ ${ }^{c}$ Sorbonne Universités, UPMC Univ. Paris 06, Institut du Calcul et de la Simulation, F-75005, Paris, France \\ ${ }^{d}$ Institut Universitaire de France, F-75005, Paris, France, \\ ${ }^{e}$ Division of Applied Mathematics, Brown University, 182 George St, Providence, RI 02912, USA \\ ${ }^{f}$ INRIA Paris-Rocquencourt, Domaine de Voluceau - Rocquencourt, B.P. 105, 78153 Le Chesnay, France
}

\begin{abstract}
In this article, we propose a post-processing of the planewave solution of the Kohn-Sham LDA model with pseudopotentials. This post-processing is based upon the fact that the exact solution can be interpreted as a perturbation of the approximate solution, allowing us to compute corrections for both the eigenfunctions and the eigenvalues of the problem in order to increase the accuracy. Indeed, this post-processing only requires the computation of the residual of the solution on a finer grid so that the additional computational cost is negligible compared to the initial cost of the planewave-based method needed to compute the approximate solution. Theoretical estimates certify an increased convergence rate in the asymptotic convergence range. Numerical results confirm the low computational cost of the post-processing and show that this procedure improves the energy accuracy of the solution even in the pre-asymptotic regime which comprises the target accuracy of practitioners.
\end{abstract}

\section{Introduction}

First-principle molecular simulation is nowadays a major tool in different fields such as chemistry, condensed matter physics and materials science. Its use is motivated by the fact that it enables one to understand and predict the properties of a molecular system, without any empirical parameter except a few fundamental constants of physics (the reduced Planck constant $\hbar$, the Boltzmann constant $k_{B}$, the mass of the electron $m_{e}$, the elementary charge $e$, the dielectric permittivity of the vacuum $\varepsilon_{0}$ ) and the masses and atomic numbers of the nuclei contained in the system under investigation.

At this level, matter is described as a system of interacting nuclei and electrons. Within the Born-Oppenheimer approximation [1] (made in almost all molecular simulations), nuclei are considered as classical point-like particles and electrons are assumed to be, at each time $t$, in their ground state. As a consequence, the nuclei dynamics is governed by a classical Hamiltonian

$$
H\left(\left\{\mathbf{R}_{k}\right\}_{1 \leq k \leq M},\left\{\mathbf{P}_{k}\right\}_{1 \leq k \leq M}\right)=\sum_{k=1}^{M} \frac{\left|\mathbf{P}_{k}\right|^{2}}{2 m_{k}}+W\left(\mathbf{R}_{1}, \mathbf{R}_{2}, \cdots, \mathbf{R}_{M}\right),
$$

where $m_{k}, \mathbf{R}_{k} \in \mathbb{R}^{3}$ and $\mathbf{P}_{k} \in \mathbb{R}^{3}$ are respectively the mass, the position and the momentum of the $k^{\text {th }}$ nucleus, where $M$ is the total number of nuclei and where $W$ is an effective potential

Email addresses: cances@cermics.enpc.fr (Eric Cancès), dusson@ann.jussieu.fr (Geneviève Dusson), maday@ann.jussieu.fr (Yvon Maday), stamm@ann.jussieu.fr (Benjamin Stamm), 
taking into account the presence of the electrons. The bottleneck in first-principle molecular simulation is the evaluation of the potential $W$ for a given nuclear configuration which requires computing the ground state energy of the electrons in the electrostatic potential generated by the nuclei. This quantity can, in principle, be computed by solving the electronic Schrödinger equation. However, as this equation is a (linear) $3 \mathcal{N}$-dimensional partial differential equation, where $\mathcal{N}$ is the number of electrons in the system, this cannot be done by brute force numerical methods when $\mathcal{N}$ exceeds two or three due to the curse of dimensionality. Different approaches have been proposed to compute the electronic ground state energy. The most popular of them can be classified in three groups:

- wavefunction methods, among which the Hartree-Fock and multiconfiguration self-consistentfield (MCSCF) models (see [4] for a mathematical introduction);

o methods originating from the density functional theory (DFT), namely orbital-free and KohnSham models, that are used and presented in details hereafter;

- quantum Monte Carlo methods $[12,13]$.

The Kohn-Sham models $[7,11]$ are the most popular approach to date as they offer a good compromise between accuracy and computational cost; they are among the most widely used models in physics and chemistry [2].

The purpose of this article is to present a new post-processing method for periodic KohnSham calculations in planewave bases, leading to a significant gain in accuracy at a very limited extra computational cost. This method is based on the observation that the exact Kohn-Sham ground state can be considered as a perturbation of the approximate Kohn-Sham ground state computed in a finite basis set, and in applying first and second-order perturbation theory to the eigenvectors and eigenvalues of the Kohn-Sham operator respectively, in order to improve their accuracies. The specific structure of the problem and the a priori error estimates in [3] allow us to identify the leading terms in these first and second-order contributions, which turn out to be easy to evaluate, and discard the other terms, which are very costly or impossible to evaluate, but negligible since proven to be small.

Our approach strongly relies on the fact that the kinetic energy operator which, from a mathematical point of view, is the leading term in the Kohn-Sham Hamiltonian commutes with the orthogonal projection on the discretization space. This is not the case for atomic orbital basis sets methods, but this is the case for other discretizations such as some wavelet methods. The extension of our approach to approximation settings that do not satisfy this commutation property requires additional theoretical investigations and is work in progress.

This article is organized as follows. We first recall in Section 2.1 the mathematical formulation of the Kohn-Sham models for isolated molecular systems. We then present the supercell KohnSham model used in condensed phase modeling and simulation (Section 2.2), and the concept of pseudopotential (Section 2.3). The planewave discretization method for the supercell Kohn-Sham model with pseudopotential is discussed in Section 3.1 and the iterative algorithms used to solve the resulting Kohn-Sham equations are detailed in Section 3.2. The a priori error estimates our analysis is based upon are reviewed in Section 3.3. We then introduce the post-processing in Section 4. For pedagogical reasons, we derive the expressions of the post-processed eigenfunctions and eigenvalues under the assumptions that all the eigenvalues of the Kohn-Sham operator are non-degenerate. The general case, as well as the proof of Theorem 4.1, which quantifies the improvement of the Kohn-Sham ground state energy obtained by the post-processing in the asymptotic regime, will be detailed in a mathematical analysis oriented paper [5]. In Section 5, we report numerical simulations on a simple system, an alanine molecule, obtained with the KSSOLV package [19], showing that our post-processing method leads to significant gain in accuracy (typically one order of magnitude on the energy) for a small extra cost (a few percent of the overall 
cost). Numerical simulations with the $\mathrm{CO}_{2}$ and the benzene molecules were also performed and led to very similar results, and therefore are not presented in this paper.

\section{DFT Kohn-Sham models}

\subsection{Introduction to Kohn-Sham models}

Throughout this article, we adopt the system of atomic units for which $\hbar=1, m_{e}=1, e=1$, $4 \pi \epsilon_{0}=1$. In this system of units, the charge of the electron is -1 and the charges of the nuclei are positive integers.

Let us first consider an isolated molecular system in vacuo, consisting of $M$ nuclei of charges $\left(z_{1}, \cdots, z_{M}\right) \in(\mathbb{N} \backslash\{0\})^{M}$ located at the positions $\left(\mathbf{R}_{1}, \cdots, \mathbf{R}_{M}\right) \in\left(\mathbb{R}^{3}\right)^{M}$ of the physical space, and of $\mathcal{N}$ electrons. The electrostatic potential generated by the nuclei and felt by the electrons is then given by

$$
V_{\text {nuc }}(\mathbf{r})=-\sum_{k=1}^{M} \frac{z_{k}}{\left|\mathbf{r}-\mathbf{R}_{k}\right|}
$$

In the spin-restricted Kohn-Sham model, the electronic state of a closed-shell system with an even number $\mathcal{N}=2 N$ of electrons is described by $N$ Kohn-Sham orbitals $\Phi=\left(\phi_{1}, \cdots, \phi_{N}\right)^{T} \in$ $\left[H^{1}\left(\mathbb{R}^{3}\right)\right]^{N}$ satisfying the orthonormality conditions

$$
\int_{\mathbb{R}^{3}} \phi_{i} \phi_{j}=\delta_{i j}, \quad \forall i, j=1, \ldots, N
$$

The associated electronic density

$$
\rho_{[\Phi]}(\mathbf{r}):=2 \sum_{i=1}^{N}\left|\phi_{i}(\mathbf{r})\right|^{2}
$$

plays a key-role in DFT. The factor 2 in the above expression accounts for the spin. Indeed, in the spin-restricted Kohn-Sham model, each orbital is occupied by two electrons, one with spin up and one with spin down. The Kohn-Sham ground state is then obtained by solving

$$
\inf \left\{\mathcal{E}_{0}^{\mathrm{KS}}(\Phi), \Phi=\left(\phi_{1}, \cdots, \phi_{N}\right)^{T} \in\left[H^{1}\left(\mathbb{R}^{3}\right)\right]^{N}, \int_{\mathbb{R}^{3}} \phi_{i} \phi_{j}=\delta_{i j}\right\}
$$

where the Kohn-Sham energy functional reads

$$
\mathcal{E}_{0}^{\mathrm{KS}}(\Phi):=\sum_{i=1}^{N} \int_{\mathbb{R}^{3}}\left|\nabla \phi_{i}\right|^{2}+\int_{\mathbb{R}^{3}} V_{\text {nuc }} \rho_{[\Phi]}+\frac{1}{2} D\left(\rho_{[\Phi]}, \rho_{[\Phi]}\right)+E_{\mathrm{xc}}\left(\rho_{[\Phi]}\right) .
$$

In the right-hand side of (3), the first term approximates the kinetic energy of the electrons, the second term accounts for the interactions between nuclei and electrons, and the third term is a (crude) approximation of the interaction between electrons. The bilinear form $D(\cdot, \cdot)$ is in fact the Coulomb energy functional in vacuo:

$$
D\left(\rho, \rho^{\prime}\right)=\int_{\mathbb{R}^{3}} \int_{\mathbb{R}^{3}} \frac{\rho(\mathbf{r}) \rho^{\prime}\left(\mathbf{r}^{\prime}\right)}{\left|\mathbf{r}-\mathbf{r}^{\prime}\right|} d \mathbf{r} d \mathbf{r}^{\prime}
$$

so that $\frac{1}{2} D\left(\rho_{[\Phi]}, \rho_{[\Phi]}\right)$ is the Coulomb energy of a classical charge distribution of density $\rho_{[\Phi]}$. The fourth term in the right-hand side of (3), called the exchange-correlation functional, is a correction term, which is essential to describe quantitatively, and sometimes even qualitatively, the physics and chemistry of the system. The exchange-correlation functional collects the errors made in the 
approximations of the kinetic energy and of the interactions between electrons by respectively the first and third terms of the Kohn-Sham functional. It follows from the Hohenberg-Kohn theorem $[10,14,15,18]$ that there exists an exact exchange-correlation functional, that is a functional of the electronic density $\rho_{[\Phi]}$ for which solving (2) provides the ground state electronic energy and density of the $\mathcal{N}$-body electronic Schrödinger equation. Unfortunately, no mathematical expression of the exchange-correlation functional amenable to numerical simulations is known. It therefore has to be approximated in practice. The local density approximation (LDA) consists in approximating the exchange-correlation functional by

$$
E_{\mathrm{xc}}\left(\rho_{[\Phi]}\right)=\int_{\mathbb{R}^{3}} e_{\mathrm{xc}}^{\mathrm{LDA}}\left(\rho_{[\Phi]}(\mathbf{r})\right) d \mathbf{r}
$$

where $e_{\mathrm{xc}}^{\mathrm{LDA}}(\bar{\rho})$ is an approximation of the exchange-correlation energy per unit volume in a homogeneous electron gas with density $\bar{\rho}$.

\subsection{Periodic Kohn-Sham models}

In the sequel, we will focus on the periodic versions of the Kohn-Sham LDA model. In the periodic setting, the nuclear configuration is supposed to be $\mathcal{R}$-periodic, where $\mathcal{R}$ is a discrete periodic lattice of $\mathbb{R}^{3}$, and the simulation domain, sometimes referred to as the supercell, no longer consists of the whole space $\mathbb{R}^{3}$ (as in (2)-(3)) but is a unit cell, denoted here by $\Omega$, of the periodic lattice $\mathcal{R}$. Periodic boundary conditions (PBC) are imposed to the Kohn-Sham orbitals (Born-von Karman PBC) at the boundary $\partial \Omega$ of the simulation cell $\Omega$. This is the standard method to compute condensed phase properties with a limited number of atoms in the simulation cell, hence at a moderate computational cost. In most applications in solid state physics and materials science, the periodic Kohn-Sham models are discretized in Fourier modes, more commonly referred to as planewave basis sets in the physics and chemistry literature. This is the reason why we focus on this particular discretization method in the present work.

As a consequence, the domain of integration in (3) and (5) is now $\Omega$ instead of $\mathbb{R}^{3}$, and the Coulomb energy is now defined as

$$
D_{\Omega}\left(\rho, \rho^{\prime}\right)=\int_{\Omega} \int_{\Omega} G_{\Omega}\left(\mathbf{r}, \mathbf{r}^{\prime}\right) \rho(\mathbf{r}) \rho^{\prime}\left(\mathbf{r}^{\prime}\right) d \mathbf{r} d \mathbf{r}^{\prime}=\int_{\Omega} \rho\left(\mathbf{r}^{\prime}\right)\left[V_{\text {coul }}\left(\rho^{\prime}\right)\right]\left(\mathbf{r}^{\prime}\right) d \mathbf{r}^{\prime}
$$

where the Green's function $G_{\Omega}$ and the periodic Coulomb potential $V_{\text {coul }}\left(\rho^{\prime}\right)$ are respectively solutions to the following problems

$$
\left\{\begin{array} { l } 
{ - \Delta G _ { \Omega } = 4 \pi ( \sum _ { \mathbf { k } \in \mathcal { R } } \delta _ { \mathbf { k } } - \frac { 1 } { | \Omega | } ) \quad \text { in } \quad \mathbb { R } ^ { 3 } , } \\
{ G _ { \Omega } \mathcal { R } - \text { periodic, } } \\
{ \int _ { \Omega } G _ { \Omega } = 0 , }
\end{array} \quad \text { and } \quad \left\{\begin{array}{l}
-\Delta V_{\text {coul }}\left(\rho^{\prime}\right)=4 \pi\left(\rho^{\prime}-\frac{1}{|\Omega|} \int_{\Omega} \rho^{\prime}\right) \text { in } \mathbb{R}^{3} \\
V_{\text {coul }}\left(\rho^{\prime}\right) \mathcal{R} \text { - periodic, } \\
\int_{\Omega} V_{\text {coul }}\left(\rho^{\prime}\right)=0
\end{array}\right.\right.
$$

The exchange-correlation functional in this periodic setting is given by

$$
E_{\mathrm{xc}, \Omega}(\rho)=\int_{\Omega} e_{\mathrm{xc}}^{\mathrm{LDA}}(\rho(\mathbf{r})) d \mathbf{r},
$$

and the orthonormality constraints read

$$
\int_{\Omega} \phi_{i} \phi_{j}=\delta_{i j}
$$




\subsection{Pseudopotentials}

The core electrons of an atom are barely affected by the chemical environment. In pseudopotential methods, the all-electron model is replaced by a reduced model explicitly dealing with valence electrons only, while core electrons are frozen in some reference state. The valence electrons are described by valence pseudo-orbitals, and the interaction between the valence electrons and the ionic cores (an ionic core consists of a nucleus and of the associated core electrons) is modelled by a nonlocal operator called a pseudopotential, constructed once and for all from single-atom reference calculations. The reduction of dimensionality obtained by getting rid of the core electrons results in a much less computationally expensive approach since only the valence pseudo-orbitals need to be computed. In addition, pseudopotentials are constructed in such a way that the valence pseudo-orbitals oscillate much less than the valence orbitals in the core region, hence can be approximated using smaller planewave bases, or discretized on coarser grids. Lastly, pseudopotentials are used to take into account, in the nonrelativistic framework of the Kohn-Sham model, some relativistic effects which play an important role in the simulation of heavy atoms.

The resulting model for the pseudo-orbitals is similar to (2)-(3), but presents some differences:

(i) $N$ now denotes the number of valence electron pairs;

(ii) $\Phi$ now denotes the set of the pseudo-orbitals of the valence electrons;

(iii) the nuclear potential $V_{\text {nuc }}$ is replaced by a pseudopotential operator $V_{\text {ion }}$ modelling the interaction between the valence electrons on the one hand, and the nuclei and the core electrons on the other hand.

More precisely, the pseudopotential consists of two terms: a local component $V_{\text {local }}$ (whose associated operator is the multiplication by the $\mathcal{R}$-periodic function $V_{\text {local }}$ ) and a nonlocal component $V_{\text {nl }}$ given by

$$
V_{\mathrm{nl}} \phi=\sum_{j=1}^{J}\left(\int_{\Omega} \xi_{j}(\mathbf{r}) \phi(\mathbf{r}) d \mathbf{r}\right) \xi_{j}
$$

where $\xi_{j}$ are regular enough $\mathcal{R}$-periodic functions and $J$ is an integer depending on the chemical nature of the ions in the unit cell. As a consequence, the second term in the Kohn-Sham energy functional (3) is replaced by

$$
\int_{\Omega} \rho_{[\Phi]} V_{\text {local }}+2 \sum_{i=1}^{N}\left\langle\phi_{i}\left|V_{\mathrm{nl}}\right| \phi_{i}\right\rangle
$$

where the Dirac bra-ket notation is used to represent the non-local part of the operator $V_{\text {ion }}$.

Further, a correction to the exchange-correlation energy due to the introduction of pseudpotential is done by setting

$$
E_{\mathrm{xc}, \Omega}^{c}\left(\rho_{[\Phi]}\right)=\int_{\Omega} e_{\mathrm{xc}}^{\mathrm{LDA}}\left(\rho_{c}(\mathbf{r})+\rho_{[\Phi]}(\mathbf{r})\right) d \mathbf{r}
$$

where $\rho_{c} \geq 0$ is a nonlinear core-correction.

To summarize, we are therefore considering the following energy functional

$$
\mathcal{E}_{0, \Omega}^{\mathrm{KS}}(\Phi)=\sum_{i=1}^{N} \int_{\Omega}\left|\nabla \phi_{i}\right|^{2}+\int_{\Omega} V_{\text {local }} \rho_{[\Phi]}+2 \sum_{i=1}^{N}\left\langle\phi_{i}\left|V_{\mathrm{nl}}\right| \phi_{i}\right\rangle+\frac{1}{2} D_{\Omega}\left(\rho_{[\Phi]}, \rho_{[\Phi]}\right)+E_{\mathrm{xc}, \Omega}^{c}\left(\rho_{[\Phi]}\right),
$$

and the set of admissible states

$$
\mathcal{M}=\left\{\Phi=\left(\phi_{1}, \cdots, \phi_{N}\right)^{T} \in\left[H_{\#}^{1}(\Omega)\right]^{N} \mid \int_{\Omega} \phi_{i} \phi_{j}=\delta_{i j}\right\}
$$


where

$$
H_{\#}^{1}(\Omega)=\left\{\phi \in H_{\mathrm{loc}}^{1}\left(\mathbb{R}^{3}\right) \mid \phi \quad \mathcal{R}-\text { periodic }\right\}
$$

is the $\mathcal{R}$-periodic $H^{1}$-space.

The ground state energy is then defined by

$$
I_{0}^{K S}=\inf \left\{\mathcal{E}_{0, \Omega}^{\mathrm{KS}}(\Phi), \Phi \in \mathcal{M}\right\} .
$$

It can be proven that, under reasonable assumptions on $V_{\mathrm{nl}}, V_{\mathrm{local}}$, and $E_{\mathrm{xc}, \Omega}^{c}$ (see [3]), (9) has a minimizer $\Phi^{0}=\left(\phi_{1}^{0}, \ldots, \phi_{N}^{0}\right) \in \mathcal{M}$ (see Section 3.3).

The first optimality conditions read

$$
\forall 1 \leq i \leq N, \quad \mathcal{H}^{0} \phi_{i}^{0}=\sum_{j=1}^{N} \lambda_{i j}^{0} \phi_{j}^{0}, \quad \int_{\Omega} \phi_{i}^{0} \phi_{j}^{0}=\delta_{i j},
$$

where the $N \times N$ matrix $\Lambda^{0}=\left(\lambda_{i j}^{0}\right)$, which is the Lagrange multiplier of the matrix constraint $\int_{\Omega} \phi_{i} \phi_{j}=\delta_{i j}$, is symmetric, and where the Hamiltonian $\mathcal{H}^{0}$ is defined as follows:

$$
\mathcal{H}^{0}=-\frac{1}{2} \Delta+V_{\text {ion }}+V_{\text {coul }}\left(\rho^{0}\right)+V_{\text {xc }}\left(\rho^{0}\right)
$$

with $\rho^{0}=\rho_{\left[\Phi^{0}\right]}, V_{\text {ion }}=V_{\text {local }}+V_{\text {nl }}$, and where

$$
V_{\mathrm{xc}}(\rho)(\mathbf{r})=\frac{d e_{\mathrm{xc}}^{\mathrm{LDA}}}{d \rho}\left(\rho_{c}(\mathbf{r})+\rho(\mathbf{r})\right) .
$$

Note that $\mathcal{H}^{0}$ is the Kohn-Sham operator

$$
\mathcal{H}_{[\rho]}=-\frac{1}{2} \Delta+V_{\text {ion }}+V_{\text {coul }}(\rho)+V_{\text {xc }}(\rho),
$$

in the case where $\rho$ is the ground state density $\rho^{0}$.

In fact, (9) has an infinity of minimizers since any unitary transform of the Kohn-Sham orbitals $\Phi^{0}$ in the sense of (12) below is also a minimizer of the Kohn-Sham energy. This is a consequence of the following invariance property:

$$
\forall \Phi \in \mathcal{M}, \quad \forall U \in \mathcal{U}(N), \quad U \Phi \in \mathcal{M}, \quad \rho_{[U \Phi]}=\rho_{[\Phi]} \quad \text { and } \quad \mathcal{E}_{0, \Omega}^{\mathrm{KS}}(U \Phi)=\mathcal{E}_{0, \Omega}^{\mathrm{KS}}(\Phi),
$$

where $\mathcal{U}(N)$ is the group of orthogonal matrices:

$$
\mathcal{U}(N)=\left\{U \in \mathbb{R}^{N \times N} \mid U^{T} U=1_{N}\right\},
$$

$1_{N}$ denoting the identity matrix of rank $N$. This invariance can be exploited to diagonalize the matrix of the Lagrange multipliers of the orthonormality constraints (see e.g. [7]), yielding the existence of a minimizer (still denoted by $\Phi^{0}$ ) with the same density $\rho^{0}$, such that

$$
\mathcal{H}^{0} \phi_{i}^{0}=\epsilon_{i}^{0} \phi_{i}^{0}, \quad \int_{\Omega} \phi_{i}^{0} \phi_{j}^{0}=\delta_{i j}
$$

for some $\epsilon_{1}^{0} \leq \epsilon_{2}^{0} \leq \cdots \leq \epsilon_{N}^{0}$.

As discussed in [3], it is not known whether Kohn-Sham ground states satisfy the so-called Aufbau principle, that is whether $\epsilon_{1}^{0}, \cdots, \epsilon_{N}^{0}$ are the lowest $N$ eigenvalues of the Kohn-Sham Hamiltonian $\mathcal{H}^{0}$. However, this property seems to be satisfied in practice for most systems, and it is always satisfied for the extended Kohn-Sham model (see [3] for details). We will assume here that the molecular system under consideration does satisfy the Aufbau principle. This allows us to solve the Kohn-Sham equations using iterative algorithms such as the one described in Section 3.2, which implicitly rely on the Aufbau principle. 


\section{Discretization and resolution of the Kohn-Sham model}

\subsection{Planewave discretization}

In order to approximate the solution of (9), we first discretize the variational set $\mathcal{M}$ using a planewave basis set. This approximation setting is the state-of-the-art method for KohnSham simulations in solid state physics and materials science. Thus the computational domain $\Omega$ equipped with periodic boundary conditions can be a cubic box, or more generally the unit cell of a periodic lattice $\mathcal{R} \subset \mathbb{R}^{3}$. The valence pseudo-orbitals are expanded in terms of the functions $e_{\mathbf{k}}(\mathbf{r}):=|\Omega|^{-1 / 2} e^{i \mathbf{k} \cdot \mathbf{r}}$, which are the Fourier modes with wavevectors $\mathbf{k} \in \mathcal{R}^{*}$, where $\mathcal{R}^{*}$ denotes the dual lattice of $\mathcal{R}$. The lattice $\mathcal{R}^{*}$ indeed consists of all wavevectors $\mathbf{k}$ such that $e_{\mathbf{k}}$ is $\mathcal{R}$-periodic. In this article, we assume for simplicity that $\Omega=[0, L)^{3}(L>0)$ but our arguments can be easily extended to the general case. In this case, $\mathcal{R}=L \mathbb{Z}^{3}$ and $\mathcal{R}^{*}=\frac{2 \pi}{L} \mathbb{Z}^{3}$.

Recall that the family $\left(e_{\mathbf{k}}\right)_{\mathbf{k} \in \mathcal{R}^{*}}$ forms an orthonormal basis of

$$
L_{\#}^{2}(\Omega, \mathbb{C}):=\left\{u \in L_{\text {loc }}^{2}\left(\mathbb{R}^{3}, \mathbb{C}\right) \mid u \text { is } \mathcal{R} \text {-periodic }\right\},
$$

and that for all $v \in L_{\#}^{2}(\Omega, \mathbb{C})$,

$$
v(\mathbf{r})=\sum_{\mathbf{k} \in \mathcal{R}^{*}} \widehat{v}_{\mathbf{k}} e_{\mathbf{k}}(\mathbf{r}) \quad \text { with } \quad \widehat{v}_{\mathbf{k}}=\left(e_{\mathbf{k}}, v\right)_{L_{\#}^{2}}=|\Omega|^{-1 / 2} \int_{\Omega} v(\mathbf{r}) e^{-i \mathbf{k} \cdot \mathbf{r}} d \mathbf{r} .
$$

For each $s \in \mathbb{R}$, we denote by

$$
H_{\#}^{s}(\Omega):=\left\{v=\left.\sum_{\mathbf{k} \in \mathcal{R}^{*}} \widehat{v}_{\mathbf{k}} e_{\mathbf{k}}\left|\forall \mathbf{k}, \quad \widehat{v}_{-\mathbf{k}}=\widehat{v}_{\mathbf{k}}^{*}, \quad\|v\|_{H_{\#}^{s}}^{2}:=\sum_{\mathbf{k} \in \mathcal{R}^{*}}\left(1+|\mathbf{k}|^{2}\right)^{s}\right| \widehat{v}_{\mathbf{k}}\right|^{2}<\infty\right\}
$$

the Sobolev space of real-valued periodic distributions with regularity $H^{s}$.

The kinetic energy of a basis function $e_{\mathbf{k}}$ is given by $\frac{1}{2}|\mathbf{k}|^{2}$, where $|\cdot|$ denotes the Euclidean norm. We introduce some energy cutoff $E_{\mathrm{c}}>0$ and consider all basis functions whose kinetic energy is smaller than $E_{\mathrm{c}}$, i.e. $|\mathbf{k}| \leq \sqrt{2 E_{\mathrm{c}}}$, to define the approximation space. That is, for each cutoff $E_{\mathrm{c}}$, we set $N_{\mathrm{c}}=\sqrt{\frac{E_{\mathrm{c}}}{2}} \frac{L}{\pi}$ and consider the finite-dimensional discretization space

$$
X_{N_{\mathrm{c}}}:=\left\{\sum_{\mathbf{k} \in \mathcal{R}^{*},|\mathbf{k}| \leq \frac{2 \pi}{L} N_{\mathrm{c}}} \widehat{v}_{\mathbf{k}} e_{\mathbf{k}} \mid \forall \mathbf{k}, \quad \widehat{v}_{-\mathbf{k}}=\widehat{v}_{\mathbf{k}}^{*}\right\} \subset \bigcap_{s \in \mathbb{R}} H_{\#}^{s}(\Omega) .
$$

We also denote by $\Pi_{N_{\mathrm{c}}}$, the linear operator on the space of $\mathcal{R}$-periodic distributions defined by

$$
\Pi_{N_{\mathrm{c}}}\left(\sum_{\mathbf{k} \in \mathcal{R}^{*}} \widehat{v}_{\mathbf{k}} e_{\mathbf{k}}\right)=\sum_{\mathbf{k} \in \mathcal{R}^{*},|\mathbf{k}| \leq \frac{2 \pi}{L} N_{\mathrm{c}}} \widehat{v}_{\mathbf{k}} e_{\mathbf{k}} .
$$

The operator $\left.\Pi_{N_{\mathrm{c}}}\right|_{H_{\#}^{s}(\Omega)}$ (which we shall also denote by $\Pi_{N_{\mathrm{c}}}$ for convenience) is in fact the orthogonal projector from $H_{\#}^{s}(\Omega)$ to $X_{N_{\mathrm{c}}}$ for any $s \in \mathbb{R}$, and we denote by $\Pi_{N_{\mathrm{c}}}^{\perp}=\left(1-\Pi_{N_{\mathrm{c}}}\right)$ the orthogonal projector on $X_{N_{\mathrm{c}}}^{\perp}$, the orthogonal of $X_{N_{\mathrm{c}}}$ in $H_{\#}^{s}(\Omega)$ (again for any $s \in \mathbb{R}$ ). Finally, the variational approximation to the ground state energy in $X_{N_{\mathrm{c}}}$ is defined as

$$
I_{0, N_{\mathrm{c}}}^{\mathrm{KS}}=\inf \left\{\mathcal{E}_{0}^{\mathrm{KS}}\left(\Phi_{N_{\mathrm{c}}}\right), \Phi_{N_{\mathrm{c}}} \in \mathcal{M} \cap\left[X_{N_{\mathrm{c}}}\right]^{N}\right\} .
$$

Using once again the invariance property (12), the Euler equations of this minimization problem can be diagonalized and therefore reduced to find the pairs $\left(\phi_{j, N_{\mathrm{c}}}, \epsilon_{j, N_{\mathrm{c}}}\right)_{j=1, \ldots, N}$ satisfying

$$
\mathcal{H}_{N_{\mathrm{c}}, \operatorname{proj}} \phi_{j, N_{\mathrm{c}}}=\epsilon_{j, N_{\mathrm{c}}} \phi_{j, N_{\mathrm{c}}}, \quad \int_{\Omega} \phi_{i, N_{\mathrm{c}}} \phi_{j, N_{\mathrm{c}}}=\delta_{i j}, \quad \epsilon_{1, N_{\mathrm{c}}} \leq \epsilon_{2, N_{\mathrm{c}}} \leq \ldots \leq \epsilon_{N, N_{\mathrm{c}}}
$$


for all $i, j=1, \ldots, N$. Here we define $\mathcal{H}_{N_{\mathrm{c}}, \operatorname{proj}}: X_{N_{\mathrm{c}}} \rightarrow X_{N_{\mathrm{c}}}$ by

$$
\mathcal{H}_{N_{\mathrm{c}}, \text { proj }}=\Pi_{N_{\mathrm{c}}} \mathcal{H}_{\left[\rho_{N_{\mathrm{c}}}\right]} \Pi_{N_{\mathrm{c}}}=-\frac{1}{2} \Pi_{N_{\mathrm{c}}} \Delta \Pi_{N_{\mathrm{c}}}+\Pi_{N_{\mathrm{c}}}\left[V_{\text {ion }}+V_{\text {coul }}\left(\rho_{N_{\mathrm{c}}}\right)+V_{\mathrm{xc}}\left(\rho_{N_{\mathrm{c}}}\right)\right] \Pi_{N_{\mathrm{c}}}
$$

with $\rho_{N_{\mathrm{c}}}=\rho_{\left[\Phi_{N_{\mathrm{c}}}\right]}, \Phi_{N_{\mathrm{c}}}=\left(\phi_{1, N_{\mathrm{c}}}, \ldots, \phi_{N, N_{\mathrm{c}}}\right)^{T}$ and where $\mathcal{H}_{\left[\rho_{N_{\mathrm{c}}}\right]}$ is defined by (11) for the approximate ground state density $\rho_{N_{\mathrm{c}}}$. A key observation is that $\mathcal{H}_{N_{\mathrm{c}} \text {,proj }}$ is the restriction to $X_{N_{\mathrm{c}}}$ of the self-adjoint operator $\mathcal{H}_{N_{\mathrm{c}}}$ on $L_{\#}^{2}(\Omega)$ with domain $H_{\#}^{2}(\Omega)$ defined by

$$
\mathcal{H}_{N_{\mathrm{c}}}=-\frac{1}{2} \Delta+\Pi_{N_{\mathrm{c}}}\left[V_{\text {ion }}+V_{\text {coul }}\left(\rho_{N_{\mathrm{c}}}\right)+V_{\text {xc }}\left(\rho_{N_{\mathrm{c}}}\right)\right] \Pi_{N_{\mathrm{c}}}
$$

and that $X_{N_{\mathrm{c}}} \oplus X_{N_{\mathrm{c}}}^{\perp}$ is a $\mathcal{H}_{N_{\mathrm{c}}}$-stable decomposition of $L_{\#}^{2}(\Omega)$. More precisely, the operator $\mathcal{H}_{N_{\mathrm{c}}}$ can be decomposed as follows:

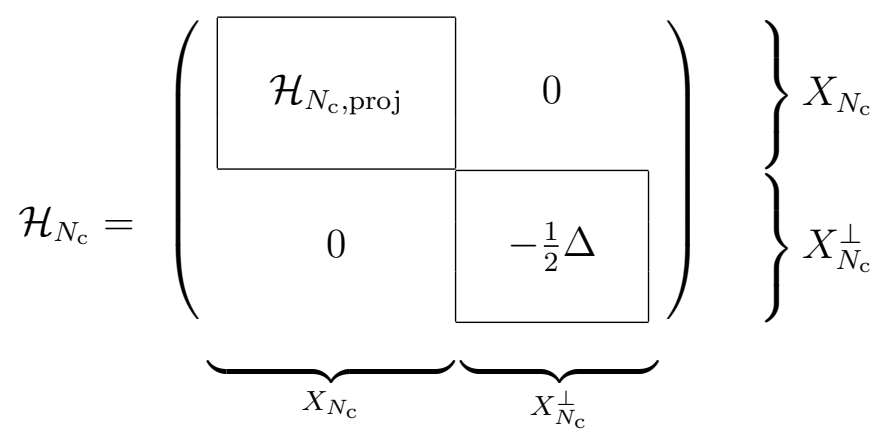

Note that, as we are using a planewave discretization, the operator $-\frac{1}{2} \Delta$ is diagonal on $X_{N_{\mathrm{c}}}^{\perp}$ with smallest eigenvalue larger than $\frac{1}{2}\left(\frac{L N_{\mathrm{c}}}{\pi}\right)^{2}$. Thus, as soon as

$$
\epsilon_{N, N_{\mathrm{c}}}<\frac{1}{2}\left(\frac{L N_{\mathrm{c}}}{\pi}\right)^{2}
$$

$\epsilon_{N, N_{\mathrm{c}}}$ being the $N^{\text {th }}$ eigenvalue of the operator $\mathcal{H}_{N_{\mathrm{c}}, \text { proj }}, \Phi_{N_{\mathrm{c}}}$ is also solution to the following eigenvalue problem

$$
\mathcal{H}_{N_{\mathrm{c}}} \phi_{j, N_{\mathrm{c}}}=\epsilon_{j, N_{\mathrm{c}}} \phi_{j, N_{\mathrm{c}}}, \quad \int_{\Omega} \phi_{i, N_{\mathrm{c}}} \phi_{j, N_{\mathrm{c}}}=\delta_{i j}, \quad \epsilon_{1, N_{\mathrm{c}}} \leq \epsilon_{2, N_{\mathrm{c}}} \leq \ldots \leq \epsilon_{N, N_{\mathrm{c}}},
$$

$\epsilon_{k, N_{\mathrm{c}}}$ being the $k$-th eigenvalue of $\mathcal{H}_{N_{\mathrm{c}}}$.

Further, the energy can then alternatively, but equivalently to (8), be obtained by

$$
\mathcal{E}_{0, \Omega}^{\mathrm{KS}}\left(\Phi_{N_{\mathrm{c}}}\right)=2 \sum_{i=1}^{N} \epsilon_{j, N_{\mathrm{c}}}-\frac{1}{2} D_{\Omega}\left(\rho_{N_{\mathrm{c}}}, \rho_{N_{\mathrm{c}}}\right)+E_{\mathrm{xc}}^{c}\left(\rho_{N_{\mathrm{c}}}\right)-\int_{\Omega} V_{\mathrm{xc}}\left(\rho_{N_{\mathrm{c}}}\right) \rho_{N_{\mathrm{c}}},
$$

where the right-hand side only depends on the eigenvalues and the electron density [9]. This energy is called double-counting energy in the following.

\subsection{SCF-iterations}

In order to solve the nonlinear eigenvalue problem (15), a Self-Consistent Field (SCF) procedure is employed [19]. It consists of solving a linear eigenvalue problem at each step, at which the Hamiltonian is computed from the density found at the previous step. Moreover, a linear charge mixing is performed in order to improve the convergence of the algorithm. The algorithm can be written as follows:

1. Initialization: Take an initial guess of the orbitals $\Phi_{N_{\mathrm{c}}}^{(0)}=\left(\phi_{1, N_{\mathrm{c}}}^{(0)}, \ldots, \phi_{N, N_{\mathrm{c}}}^{(0)}\right)^{T}$ with associated density $\rho_{N_{\mathrm{c}}}^{(0)}=\rho_{\left[\Phi_{N_{\mathrm{c}}}^{(0)}\right]}$, a memory parameter $m \in \mathbb{N}$ and a tolerance $\eta>0$. 
2. Iterations: For $i=1,2, \ldots$ until convergence

(a) Compute the Hamiltonian $\mathcal{H}_{N_{\mathrm{c}}, \text { proj }}^{(i-1)}:=\Pi_{N_{\mathrm{c}}} \mathcal{H}_{\left[\rho_{N_{\mathrm{c}}}^{(i-1)}\right]} \Pi_{N_{\mathrm{c}}}$.

(b) Solve the linear eigenvalue problem

$$
\mathcal{H}_{N_{\mathrm{c}}, \operatorname{proj}}^{(i-1)} \phi_{j, N_{\mathrm{c}}}^{(i)}=\epsilon_{j, N_{\mathrm{c}}}^{(i)} \phi_{j, N_{\mathrm{c}}}^{(i)}, \quad \int_{\Omega} \phi_{j, N_{\mathrm{c}}}^{(i)} \phi_{k, N_{\mathrm{c}}}^{(i)}=\delta_{i k},
$$

for $j=1, \ldots, N$ to obtain a set of orbitals $\Phi_{N_{\mathrm{c}}}^{(i)}=\left(\phi_{1, N_{\mathrm{c}}}^{(i)}, \ldots, \phi_{N, N_{\mathrm{c}}}^{(i)}\right)^{T}$, by selecting the lowest $N$ eigenvalues $\epsilon_{1, N_{\mathrm{c}}}^{(i)}, \epsilon_{2, N_{\mathrm{c}}}^{(i)}, \ldots, \epsilon_{N, N_{\mathrm{c}}}^{(i)}$, counted with their multiplicities, and corresponding eigenfunctions $\left(\phi_{1, N_{\mathrm{c}}}^{(i)}, \phi_{2, N_{\mathrm{c}}}^{(i)}, \ldots, \phi_{N, N_{\mathrm{c}}}^{(i)}\right)^{T} \in \mathcal{M}$, following the Aufbau principle.

(c) Compute the new density $\check{\rho}_{N_{\mathrm{c}}}^{(i)}=\rho_{\left[\Phi_{N_{\mathrm{c}}}^{(i)}\right]}$.

(d) Charge mixing: replace the charge density $\check{\rho}_{N_{\mathrm{c}}}^{(i)}$ with a linear combination of previously computed charge densities, i.e.,

$$
\rho_{N_{\mathrm{c}}}^{(i)}=\alpha_{0} \check{\rho}_{N_{\mathrm{c}}}^{(i)}+\sum_{k=1}^{\min (i, m)} \alpha_{k} \rho_{N_{\mathrm{c}}}^{(i-k)}
$$

with appropriately chosen mixing parameters satisfying $\sum_{k=0}^{\min (i, m)} \alpha_{k}=1$.

3. Output: If $\left\|\rho_{N_{\mathrm{c}}}^{(i)}-\rho_{N_{\mathrm{c}}}^{(i-1)}\right\|<\eta$, where $\|$.$\| is a given norm, stop, else go back to step 2$.

Note that several points in this algorithm need to be specified for its practical implementation. Indeed, several linear eigenvalue solvers and several charge mixing procedures are available, and different norms can be used for the convergence test. Every possible choice could be used, without affecting the results presented in the next section. The choices made for the numerical tests reported in Section 5 will be described there.

\subsection{Smoothness assumptions and a priori results}

In order to guarantee the existence of minimizers of problem (9) and to study the convergence of the solutions to the discretized problem (14) to those of the continuous problem (9), some assumptions on the data are needed. However, to avoid technicalities, these assumptions are not detailed in the present paper and the interested reader is referred to [3].

First, under sufficient regularity assumptions, problem (9) with energy functional (8) has a minimizer $\Phi^{0}$ satisfying (13). Note that the $\mathrm{X} \alpha$ exchange-correlation functional defined by $e_{\mathrm{xc}}^{\mathrm{X} \alpha}(\rho)=-C_{\mathrm{X}} \rho^{4 / 3}$, where $C_{\mathrm{X}}>0$ is a given constant, satisfies these assumptions. They are also satisfied by the exact exchange-correlation functional.

Second, the following a priori estimates hold.

Theorem 3.1 ([3]). Under sufficient regularity assumptions, there exists $r^{0}>0$ and $N_{\mathrm{c}}^{0}$ such that for $N_{\mathrm{c}} \geq N_{\mathrm{c}}^{0}$, (14) has a unique local minimizer $\Phi_{N_{\mathrm{c}}}^{0}$ in the set

$$
\left\{\Phi_{N_{\mathrm{c}}} \in\left(X_{N_{\mathrm{c}}}\right)^{N} \cap \mathcal{M}^{\Phi^{0}} \mid\left\|\Phi_{N_{\mathrm{c}}}-\Phi^{0}\right\|_{H_{\#}^{1}} \leq r^{0}\right\}
$$

with

$$
\mathcal{M}^{\Phi^{0}}=\left\{\Psi \in \mathcal{M} \mid\left\|\Psi-\Phi^{0}\right\|_{L_{\#}^{2}}=\min _{U \in \mathcal{U}(N)}\left\|U \Psi-\Phi^{0}\right\|_{L_{\#}^{2}}\right\}
$$


where $\mathcal{U}(N)$ is the set of all unitary transforms in $\mathbb{R}^{N}$. Besides,

$$
\begin{gathered}
\left\|\Phi_{N_{\mathrm{c}}}^{0}-\Phi^{0}\right\|_{H_{\#}^{s}} \leq C_{s} N_{\mathrm{c}}^{-(2-s)}\left\|\Pi_{N_{\mathrm{c}}} \Phi^{0}-\Phi^{0}\right\|_{H_{\#}^{2}}, \\
\left|\epsilon_{i, N_{\mathrm{c}}}^{0}-\epsilon_{i}^{0}\right| \underset{N_{\mathrm{c}} \rightarrow \infty}{\longrightarrow} 0, \\
\gamma\left\|\Phi_{N_{\mathrm{c}}}^{0}-\Phi^{0}\right\|_{H_{\#}^{1}}^{2} \leq I_{0, N_{\mathrm{c}}}^{\mathrm{KS}}-I_{0}^{\mathrm{KS}} \leq C\left\|\Phi_{N_{\mathrm{c}}}^{0}-\Phi^{0}\right\|_{H_{\#}^{1}}^{2},
\end{gathered}
$$

for all $0 \leq s \leq 2$, and for some constants $\gamma>0, C_{s} \geq 0$, and $C \geq 0$ independent of $N_{c}$, where the $\epsilon_{i}^{0}, \epsilon_{i, N_{\mathrm{c}}}^{0}, 1 \leq i \leq N$, are the $N$ lowest eigenvalues (counting multiplicities) of the Hamiltonians $\mathcal{H}^{0}=H_{\left.\rho_{[\Phi} 0\right]}$ and $\mathcal{H}_{N_{\mathrm{c}}}=H_{\rho_{\left[\Phi_{N_{\mathrm{c}}}\right]}}$ respectively.

Stronger results can be obtained under additional assumptions on the exchange-correlation functional. We refer to [3] for the details.

\section{A post-processing based on perturbation theory}

In this section, we propose a post-processing that is based upon the fact that the exact solution of the Kohn-Sham problem can be interpreted as a perturbation of the approximate solution. We assume that the tolerance parameter $\eta$ defined in the SCF-procedure is sufficiently small. Given the result of the converged SCF procedure, i.e. given the eigenfunctions $\Phi_{N_{\mathrm{c}}}=\left(\phi_{1, N_{\mathrm{c}}}, \ldots, \phi_{N, N_{\mathrm{c}}}\right)^{T}$ and eigenvalues $\left(\epsilon_{j, N_{c}}\right)_{j=1, \ldots, N}$ with density $\rho_{N_{c}}$ of the discretized nonlinear eigenvalue problem in the space $X_{N_{\mathrm{c}}}$, it is then possible to compute corrections for both the eigenfunctions and the eigenvalues of the problem in order to increase the accuracy.

The key observation is that the exact solution $\left(\phi_{j}^{0}, \epsilon_{j}^{0}\right)_{j=1, \ldots, N}$ satisfies

$$
\left(\mathcal{H}_{N_{\mathrm{c}}}+\mathcal{V}_{N_{\mathrm{c}}}+\mathcal{W}_{N_{\mathrm{c}}}\right) \phi_{j}^{0}=\epsilon_{j}^{0} \phi_{j}^{0}, \quad \int_{\Omega} \phi_{i}^{0} \phi_{j}^{0}=\delta_{i j}
$$

where

$$
\begin{aligned}
\mathcal{V}_{N_{\mathrm{c}}} & =\left[V_{\text {ion }}+V_{\text {coul }}\left(\rho_{N_{\mathrm{c}}}\right)+V_{\mathrm{xc}}\left(\rho_{N_{\mathrm{c}}}\right)\right]-\Pi_{N_{\mathrm{c}}}\left[V_{\text {ion }}+V_{\text {coul }}\left(\rho_{N_{\mathrm{c}}}\right)+V_{\mathrm{xc}}\left(\rho_{N_{\mathrm{c}}}\right)\right] \Pi_{N_{\mathrm{c}}}, \\
\mathcal{W}_{N_{\mathrm{c}}} & =\left[V_{\text {coul }}\left(\rho^{0}\right)+V_{\mathrm{xc}}\left(\rho^{0}\right)\right]-\left[V_{\text {coul }}\left(\rho_{N_{\mathrm{c}}}\right)+V_{\mathrm{xc}}\left(\rho_{N_{\mathrm{c}}}\right)\right] .
\end{aligned}
$$

With these definitions, we obtain that

$$
\mathcal{H}_{\left[\rho_{N_{\mathrm{c}}}\right]}=\mathcal{H}_{N_{\mathrm{c}}}+\mathcal{V}_{N_{\mathrm{c}}} \quad \text { and } \quad \mathcal{H}^{0}=\mathcal{H}_{N_{\mathrm{c}}}+\mathcal{V}_{N_{\mathrm{c}}}+\mathcal{W}_{N_{\mathrm{c}}}
$$

We then apply the nonlinear Rayleigh-Schrödinger perturbation method (see e.g. [8] and [6] for a mathematical analysis) using $\left(\phi_{j, N_{\mathrm{c}}}, \epsilon_{j, N_{\mathrm{c}}}\right)_{j=1, \ldots, N}$ as the reference solution and $\left(\phi_{j}^{0}, \epsilon_{j}^{0}\right)_{j=1, \ldots, N}$ as the perturbed solution, in order to build improved approximations $\left(\widetilde{\phi}_{j, N_{\mathrm{c}}}, \widetilde{\epsilon}_{j, N_{\mathrm{c}}}\right)_{j=1, \ldots, N}$ based upon perturbation arguments. The use of perturbation theory in this setting seems to be new.

For the sake of simplicity, we will explain the argument assuming that all the eigenvalues under consideration are simple, so that non-degenerate perturbation theory applies. The general case can be dealt with the density matrix formalism (see [5]).

Since $\mathcal{V}_{N_{\mathrm{c}}}+\mathcal{W}_{N_{\mathrm{c}}}$ is a compact perturbation of the Hamiltonian $\mathcal{H}_{N_{\mathrm{c}}}$, we can define for $\nu \in[0,1]$ $\mathcal{H}(\nu)=\mathcal{H}_{N_{\mathrm{c}}}+\nu\left(\mathcal{V}_{N_{\mathrm{c}}}+\mathcal{W}_{N_{\mathrm{c}}}\right)$ so that $\mathcal{H}(0)=\mathcal{H}_{N_{\mathrm{c}}}$ and $\mathcal{H}(1)=\mathcal{H}^{0}$. Expanding the orbitals and eigenvalues in terms of powers of the perturbation parameter $\nu$ and applying Kato's regular perturbation theory results in

$$
\phi_{j}(\nu)=\sum_{\ell=0}^{\infty} \nu^{\ell} \phi_{j, N_{\mathrm{c}}}^{(\ell)}, \quad \epsilon_{j}(\nu)=\sum_{\ell=0}^{\infty} \nu^{\ell} \epsilon_{j, N_{\mathrm{c}}}^{(\ell)}, \quad \text { for all } \quad j=1, \ldots, N,
$$


so that (taking $\nu=1$ )

$$
\phi_{j}^{0}=\sum_{\ell=0}^{\infty} \phi_{j, N_{\mathrm{c}}}^{(\ell)}, \quad \epsilon_{j}^{0}=\sum_{\ell=0}^{\infty} \epsilon_{j, N_{\mathrm{c}}}^{(\ell)}, \quad \text { for all } \quad j=1, \ldots, N,
$$

with $\left(\phi_{j, N_{\mathrm{c}}}^{(0)}, \epsilon_{j, N_{\mathrm{c}}}^{(0)}\right)=\left(\phi_{j, N_{\mathrm{c}}}, \epsilon_{j, N_{\mathrm{c}}}\right)$ being the solution of the unperturbed $(\nu=0)$ nonlinear eigenvalue problem (17). Indeed, one can show [5] that $\mathcal{V}_{N_{\mathrm{c}}}+\mathcal{W}_{N_{\mathrm{c}}}$ is not only $\mathcal{H}_{N_{\mathrm{c}}}$-bounded but that the bound tends to 0 when $N_{\mathrm{c}} \rightarrow+\infty$. In consequence, the convergence radii of the above series tend to infinity when $N_{\mathrm{c}}$ increases, so that we can guarantee convergence for $\nu=1$ and $N_{\mathrm{c}}$ sufficiently large.

Further, incorporating a priori results stating that the $H^{1}$-norm of the first-order perturbation of the orbitals generated by $\mathcal{W}_{N_{\mathrm{c}}}$ is negligible with respect to the one generated by $\mathcal{V}_{N_{\mathrm{c}}}$ allows us to consider only the latter, called $\phi_{j, N_{\mathrm{c}}}^{(1,1)}$. Then, a simple calculation shows that the first-order correction to the eigenfunctions $\left(\phi_{j, N_{\mathrm{c}}}\right)_{j=1, \ldots, N}$ due to $\mathcal{V}_{N_{\mathrm{c}}}$ is well-defined provided that equation (16) is verified, and is given by (see [5] for details)

$$
\phi_{j, N_{\mathrm{c}}}^{(1,1)}=-\left(-\left.\frac{1}{2} \Delta\right|_{X_{N_{\mathrm{c}}}^{\perp}}-\epsilon_{j, N_{\mathrm{c}}}\right)^{-1} r_{j} \quad \text { for all } j=1, \ldots, N,
$$

where

$$
r_{j}=\mathcal{H}_{\left[\rho_{N_{\mathrm{c}}}\right]} \phi_{j, N_{\mathrm{c}}}-\epsilon_{j, N_{\mathrm{c}}} \phi_{j, N_{\mathrm{c}}}=\left(\mathcal{H}_{N_{\mathrm{c}}}+\mathcal{V}_{N_{\mathrm{c}}}\right) \phi_{j, N_{\mathrm{c}}}-\epsilon_{j, N_{\mathrm{c}}} \phi_{j, N_{\mathrm{c}}} \in X_{N_{\mathrm{c}}}^{\perp},
$$

is the residual of the eigenvalue problem, which can also be written as

$$
r_{j}=\left(-\frac{1}{2} \Delta+V_{\text {ion }}+V_{\text {coul }}\left(\rho_{N_{\mathrm{c}}}\right)+V_{\mathrm{xc}}\left(\rho_{N_{\mathrm{c}}}\right)-\epsilon_{j, N_{\mathrm{c}}}\right) \phi_{j, N_{\mathrm{c}}} .
$$

Note that as $\phi_{j, N_{\mathrm{c}}}^{(1,1)} \in X_{N_{\mathrm{c}}}^{\perp}$, and $-\left(-\left.\frac{1}{2} \Delta\right|_{X_{N_{\mathrm{c}}}^{\perp}}-\epsilon_{j, N_{\mathrm{c}}}\right)^{-1}$ is a diagonal operator in the Fourier basis, the first-order correction to the $j$-th eigenvector $\phi_{j, N_{\mathrm{c}}}^{(1,1)}$ is easy to compute.

The first order correction to the eigenvalue originating from $\mathcal{V}_{N_{\mathrm{c}}}$ vanishes, and the one originating from $\mathcal{W}_{N_{\mathrm{c}}}$ is small from a priori results. The computable part of the second order correction is (using Dirac's bra-ket notation)

$$
\epsilon_{j, N_{\mathrm{c}}}^{(2,1)}=\left(\phi_{j, N_{\mathrm{c}}}^{(1,1)}, r_{j}\right)_{L_{\#}^{2}}=-\left\langle r_{j}\left|\left(-\left.\frac{1}{2} \Delta\right|_{X_{N_{\mathrm{c}}}^{\perp}}-\epsilon_{j, N_{\mathrm{c}}}\right)^{-1}\right| r_{j}\right\rangle
$$

Hence $\phi_{j, N_{\mathrm{c}}}^{(1,1)}$ and $\epsilon_{j, N_{\mathrm{c}}}^{(2,1)}$ are well-defined (provided that $N_{\mathrm{c}}$ is large enough).

We then define the post-processed solution as

$$
\widetilde{\phi}_{j, N_{\mathrm{c}}}=\phi_{j, N_{\mathrm{c}}}+\phi_{j, N_{\mathrm{c}}}^{(1,1)}, \quad \widetilde{\epsilon}_{j, N_{\mathrm{c}}}=\epsilon_{j, N_{\mathrm{c}}}+\epsilon_{j, N_{\mathrm{c}}}^{(2,1)}, \quad \text { and } \quad \widetilde{\rho}_{N_{\mathrm{c}}}=\rho_{\left[\widetilde{\Phi}_{N_{\mathrm{c}}}\right]},
$$

with $\widetilde{\Phi}_{N_{\mathrm{c}}}=\left(\widetilde{\phi}_{1, N_{\mathrm{c}}}, \ldots, \widetilde{\phi}_{N, N_{\mathrm{c}}}\right)^{T}$. Therefore, the post-processed ground state energy can be provided, either following (8) by

$$
\tilde{I}_{0, N_{\mathrm{c}}}^{\mathrm{KS}}=\sum_{i=1}^{N} \int_{\Omega}\left|\nabla \widetilde{\phi}_{i, N_{\mathrm{c}}}\right|^{2}+\int_{\Omega} V_{\text {local }} \widetilde{\rho}_{N_{\mathrm{c}}}+2 \sum_{i=1}^{N}\left\langle\widetilde{\phi}_{i, N_{\mathrm{c}}}\left|V_{\mathrm{nl}}\right| \widetilde{\phi}_{i, N_{\mathrm{c}}}\right\rangle+\frac{1}{2} D_{\Omega}\left(\widetilde{\rho}_{N_{\mathrm{c}}}, \widetilde{\rho}_{N_{\mathrm{c}}}\right)+E_{\mathrm{xc}, \Omega}^{c}\left(\widetilde{\rho}_{N_{\mathrm{c}}}\right),
$$

or following the double counting formula (18) by

$$
\tilde{I}_{0, N_{\mathrm{c}}}^{\mathrm{KS}, \mathrm{DC}}=2 \sum_{i=1}^{N} \widetilde{\epsilon}_{j, N_{\mathrm{c}}}-\frac{1}{2} D_{\Omega}\left(\widetilde{\rho}_{N_{\mathrm{c}}}, \widetilde{\rho}_{N_{\mathrm{c}}}\right)+E_{\mathrm{xc}}^{c}\left(\widetilde{\rho}_{N_{\mathrm{c}}}\right)-\int_{\Omega} V_{\mathrm{xc}}\left(\widetilde{\rho}_{N_{\mathrm{c}}}\right) \widetilde{\rho}_{N_{\mathrm{c}}} .
$$

Even though definitions (8) and (18) are equivalent for the discrete ground states, i.e. for the exact solutions to problem (17), the two perturbed energies computed by (22) and (23) are not 
equal, and lead to different numerical results. From a computational viewpoint, the time needed to compute two energies (22) and (23) is almost the same. But it seems that (22) gives better approximations for the energy than the double counting formula (23). The numerical performance of these two formulas will be discussed in Section 5 .

From a theoretical viewpoint, we can state the following result for the perturbed energy (22).

Theorem $4.1([5])$. Let $I_{0, N_{\mathrm{c}}}^{\mathrm{KS}}$ be the planewave approximation of the ground state energy be defined by (14) and $\tilde{I}_{0, N_{\mathrm{c}}}^{\mathrm{KS}}$ the post-processed approximation given by (22). Then, under sufficient regularity assumptions (see [5]), there exists a constant $C>0$, independent of $N_{\mathrm{c}}$, such that

$$
\left|\tilde{I}_{0, N_{\mathrm{c}}}^{\mathrm{KS}}-I_{0}^{\mathrm{KS}}\right| \leq C N_{\mathrm{c}}^{-2}\left|I_{0, N_{\mathrm{c}}}^{\mathrm{KS}}-I_{0}^{\mathrm{KS}}\right|,
$$

where $I_{0}^{\mathrm{KS}}$ is the exact ground state energy, defined in (9).

Although the above inequality might not be not optimal (some numerical results seem to show that the improvement factor is better than $N_{\mathrm{c}}^{-2}$ ), this result clearly indicates that the perturbation method leads to a substantial improvement of the accuracy of the energy in the asymptotic regime,

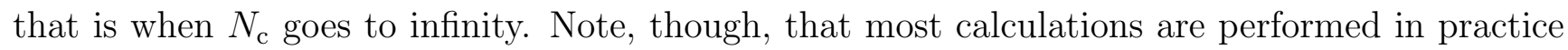
in the pre-asympotic regime, with moderate values of $N_{\mathrm{c}}$. It is therefore important to check numerically that the perturbation method performs well also in the pre-asymptotic regime, which will be done in the next section. Let us also mention that the above inequality is concerned with the energy only. Obtaining similar estimates for other properties, and in particular for atomic forces, is a difficult task, which is work in progress.

From an implementational viewpoint, the residual $r_{j}$, defined in (20), which is an infinitedimensional object belonging to $\Pi_{N_{\mathrm{c}}}^{\perp}$, is represented on a discrete space $X_{N_{\mathrm{c}} \text {,res }}$ based on some $E_{\mathrm{c}, \text { res }} \geq E_{\mathrm{c}}$ which, in turn, corresponds to a certain $N_{\mathrm{c}, \text { res }} \geq N_{\mathrm{c}}$. Further, observing that the density $\rho_{N_{\mathrm{c}}}$ belongs to $X_{2 N_{\mathrm{c}}}$ (corresponding to $4 E_{\mathrm{c}}$ and $2 N_{\mathrm{c}}$ ), the potential $V_{\text {coul }}\left(\rho_{N_{\mathrm{c}}}\right)$ therefore belongs to $X_{2 N_{\mathrm{c}}}$ as well. The post-processing requires that the potentials $V_{\text {ion }}$ and $V_{\mathrm{xc}}\left(\rho_{N_{\mathrm{c}}}\right)$ can be expressed in the larger space with cutoff $E_{\mathrm{c}, \mathrm{res}}$ so that full knowledge of all the modes of the residual lying in $X_{N_{\mathrm{c}} \text {,res }}$ are accessible. In practice, it might be simpler to obtain $V_{\text {ion }}$ and $V_{\mathrm{xc}}\left(\rho_{N_{\mathrm{c}}}\right)$ as elements of $X_{N_{\mathrm{c}} \text {,res }}$ directly in order to avoid too many data structures associated with different cutoffs. The computation of this residual requires additional Fast Fourier Transforms (FFT) on the finer grid corresponding to $E_{\text {cut,res. }}$. Indeed, applying the Hamiltonian $\mathcal{H}_{\left[\rho_{N_{\mathrm{c}}}\right]}$ to the orbitals $\phi_{j, N_{\mathrm{c}}}$ requires two additional FFT's on the fine grid for each orbital.

\section{Numerical results}

We present here some results on a small molecule as a proof of concept. The alanine molecule which has 18 valence electron pairs is considered. The computation of the planewave approximation is based on KSSOLV [19], a Matlab toolbox for solving the Kohn-Sham equations. Troullier-Martins pseudopotentials [17] are considered, in combination with the Perdew-Zunger (PZ81) LDA-functional $[11,16]$. Note that we obtained qualitatively the same results with the $\mathrm{CO}_{2}$ molecule as well as the benzene molecule.

In all what follows, the computed solutions are compared to a reference solution, which is a solution computed on a very fine grid with a kinetic energy cutoff $E_{\mathrm{c}}^{\mathrm{ref}}$ of 400 atomic units (a.u.). A coarse solution (labelled "without perturbation") is computed on a grid with cutoff $E_{\mathrm{c}}$, and the post-processed approximations given by the perturbation theory are computed on a grid with fine cutoff $E_{\text {c,res }}$ (labelled "with perturbation") (21). Note that the components of the Kohn-Sham orbitals on the coarse grid are not modified by the post-processing. The coefficients computed through the perturbation process correspond to basis functions with wave numbers larger than $E_{\mathrm{c}}$. We denote by $\lambda$ the relation between the coarse and fine cutoffs, i.e. $\lambda=\frac{E_{\mathrm{c}, \mathrm{res}}}{E_{\mathrm{c}}}$. 
In the following, we simulate three scenarios. In each case, we compare the errors on the energies of the solutions with perturbation computed with either formula (22) or formula (23) to the errors on the energies of the solutions without perturbation. First, we fix a coarse cutoff $E_{\mathrm{c}}$, and we vary the fine cutoff $E_{\mathrm{c}, \mathrm{res}}$, which corresponds to varying the parameter $\lambda$. In the second case, we fix a fine cutoff $E_{\mathrm{c} \text {,res }}$ and we vary the coarse cutoff $E_{\mathrm{c}}$, which also corresponds to varying the parameter $\lambda$. Finally, we fix $\lambda$ and we vary simultaneously the cutoffs $E_{\mathrm{c}}$ and $E_{\mathrm{c}, \text { res }}$. Thus we can observe the improvement in the energy when applying the perturbation theory in these different frameworks. This enables us to find the best compromises between accuracy and computational resources, both in time and memory.

\subsection{Simulations with a constant $E_{\mathrm{c}}$}

Here, we fix $E_{\mathrm{c}}$, hence the dimension of the space in which we compute the coarse solution. We then compute the post-processed corrections in spaces of different sizes characterized by different $E_{\text {c,res }}$ to obtain a more accurate solution. We observe on Figure 1 that for all the tested $E_{\mathrm{c}, \mathrm{res}}$, the energy of the solution with perturbative corrections is more precise than the coarse solution as the relative energy error is improved by one to two orders of magnitude, depending on the energy formula used to compute the perturbed energy. Indeed, the formula (22) gives an energy always closer to the exact energy than the formula (23) based on double counting.

The relative time increases when the parameter $\lambda$ increases, as it is more expensive to compute the perturbed solution on a larger grid than on a smaller one. However, the time necessary to compute the perturbative corrections corresponds approximately to $1 \%$ to $12 \%$ of the cost to compute the coarse solution, which is indeed very little. The cost to compute the perturbed energy is a little higher for the energy based on (22) than for the double counting energy (23), but the difference in negligible compared to the total computing time. Note that the cost in memory is also higher when $E_{\mathrm{c} \text {,res }}$ is large, as the perturbed solution is then computed on a larger grid.

The improvement of the solution seems to be constant for $\lambda \geq 3$, which means that the main improvement of the post-processing is due to coefficients corresponding to planewaves with kinetic energy slightly larger than $E_{\mathrm{c}}$. We therefore conclude that it seems useless to use a large $\lambda$ and hence a large $E_{\mathrm{c} \text {,res }}$, to post-process the solution as the improvement is negligible while the cost in time and memory increases. The best choice of $\lambda$ seems to vary, but a good choice seems to be around 2 .

\subsection{Simulations with a constant $E_{\mathrm{c}, \mathrm{res}}$}

In this case, we fix the fine grid used for the computation of the perturbed solution but we compute it from different coarse solutions obtained with different values of $E_{\mathrm{c}}$. This corresponds to a case where the limiting parameter is the memory, and so the fine grid in which we can compute the perturbed solution is fixed. It is shown on Figure 2 that it is possible to get the same accuracy in energy by doing a small computation on a coarse grid and then post-processing the coarse solution on a grid with parameter $E_{\mathrm{c}, \text { res }}$. The computational cost is in this case much reduced if we compare it to a classical approach consisting of computing the solution on the grid with cutoff $E_{\mathrm{c}, \text { res }}$.

First, the computational time needed to compute the perturbed solution from the unperturbed one is once again negligible compared to the time needed to compute the coarse solution, at least for small values of the parameter $\lambda$. The cost in terms of CPU time is also negligible using either (22) or (23) for the post-processed energy computation.

Second, we observe that for $E_{\mathrm{c}, \mathrm{res}}=100$ a.u. and $E_{\mathrm{c}, \mathrm{res}}=200$ a.u., the relative energy error for the solution with perturbation is always smaller than for the solution without perturbation by a factor of about 10 to 50. When $\lambda$ increases, the error becomes larger, which is expected since $E_{\mathrm{c}}$ decreases. Moreover it seems that the improvement is better for $E_{\mathrm{c} \text {,res }}=200$ than for $E_{\mathrm{c}, \mathrm{res}}=100$. 

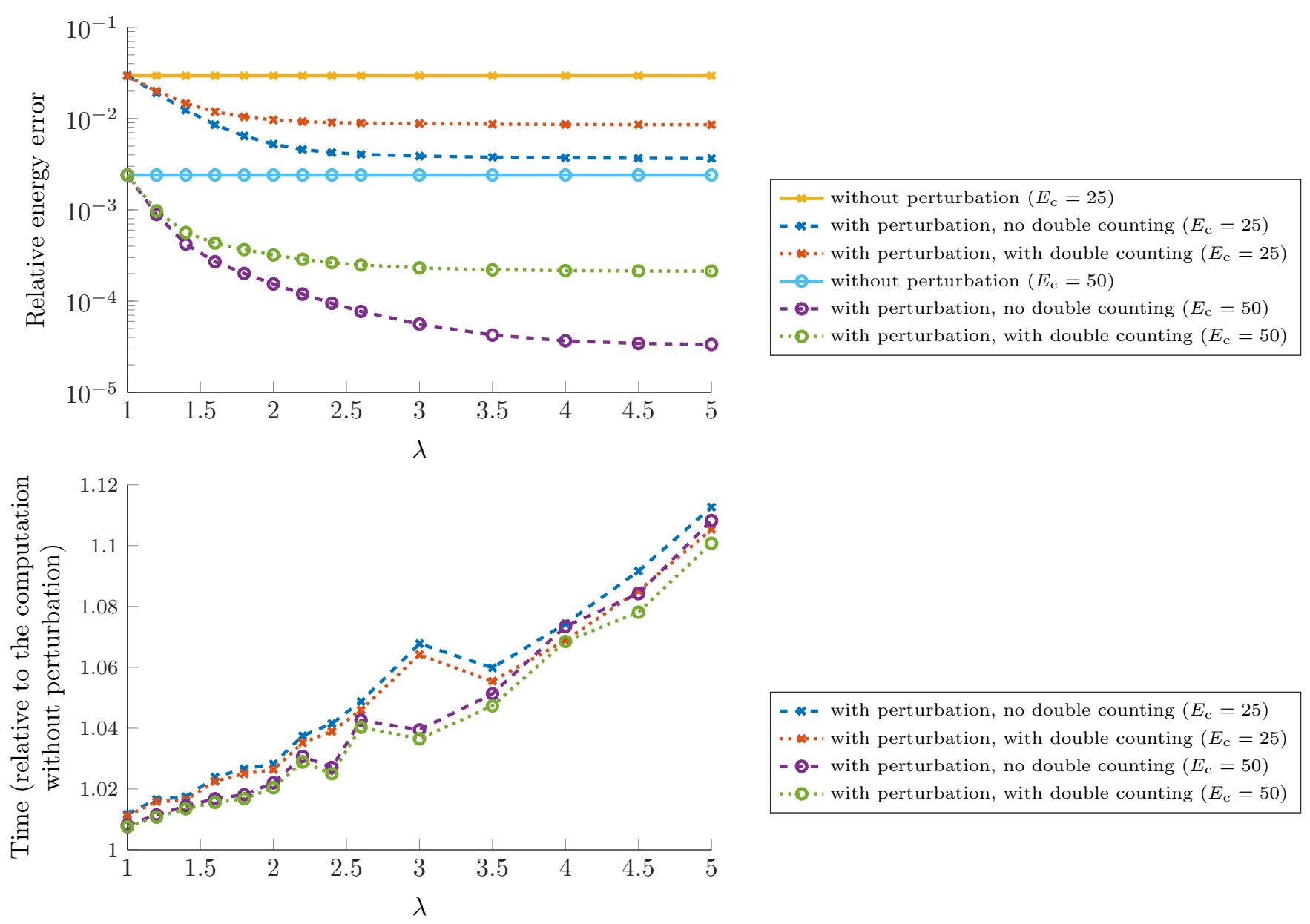

- - with perturbation, no double counting $\left(E_{\mathrm{c}}=25\right)$
$-\cdots$ with perturbation, with double counting $\left(E_{\mathrm{c}}=25\right)$
- 0 - with perturbation, no double counting $\left(E_{\mathrm{c}}=50\right)$
$=-$ with perturbation, with double counting $\left(E_{\mathrm{c}}=50\right)$

Figure 1: Simulations with a constant $E_{\mathrm{c}}$ (alanine molecule).

The post-processed energy $\tilde{I}_{0, N_{\mathrm{c}}}^{\mathrm{KS}}$ given by $(22)$ is in this case always closer to the exact energy than the energy $\tilde{I}_{0, N_{\mathrm{c}}}^{\mathrm{KS}, \mathrm{DC}}$ given by (23). Indeed, the energy error for $\tilde{I}_{0, N_{\mathrm{c}}}^{\mathrm{KS} \text {,DC }}$ is often 2 to 5 times larger than the energy error based on $\tilde{I}_{0, N_{\mathrm{c}}}^{\mathrm{KS}}$. Hence, the post-processed energy $\tilde{I}_{0, N_{\mathrm{c}}}^{\mathrm{KS}}$ improves the energy error of the solution on the coarse grid by a factor 10 to 100 whereas the post-processed energy based on double counting formula (23) improves the energy error only by a factor between 4 and 12.

Moreover, it is interesting to compare the time needed to reach a given accuracy for the method with or without perturbation. For example, for $\lambda=3$ and $E_{\mathrm{c}, \text { res }}=200$ a.u., the time needed to compute the perturbed solution is about 2600 seconds which is 7 times less than the time needed to compute the self-consistent solution on this grid (corresponding to $\lambda=1$ ), whereas the energy error $\tilde{I}_{0, N_{\mathrm{c}}}^{\mathrm{KS}}$ given by (22) is of the same order of magnitude. Hence, when the solution on the coarse grid is not too crude, the perturbation theory enables us to significantly improve the solution at very low extra cost.

\subsection{Simulations with a constant $\lambda$}

In this case, we fix the proportionality constant between $E_{\mathrm{c}}$ and $E_{\mathrm{c}, \mathrm{res}}$. For different values of $E_{\mathrm{c}}$, we consider the relative energy error without or with the perturbation corrections (Figure 3).

For all $E_{\mathrm{c}}$ greater than 20 a.u., there is an improvement in energy when performing the perturbation corrections, using one or the other energy formula. The relative energy error of the solutions with perturbation is indeed lower than for the solutions without perturbation by a factor between 2 and 100. Once again, the energy formula (22) gives better results than the formula (23). The computational time increases as $E_{\mathrm{c}}$ increases and the time necessary to compute the solution with perturbation is still very small compared to the computation of the coarse solution, 

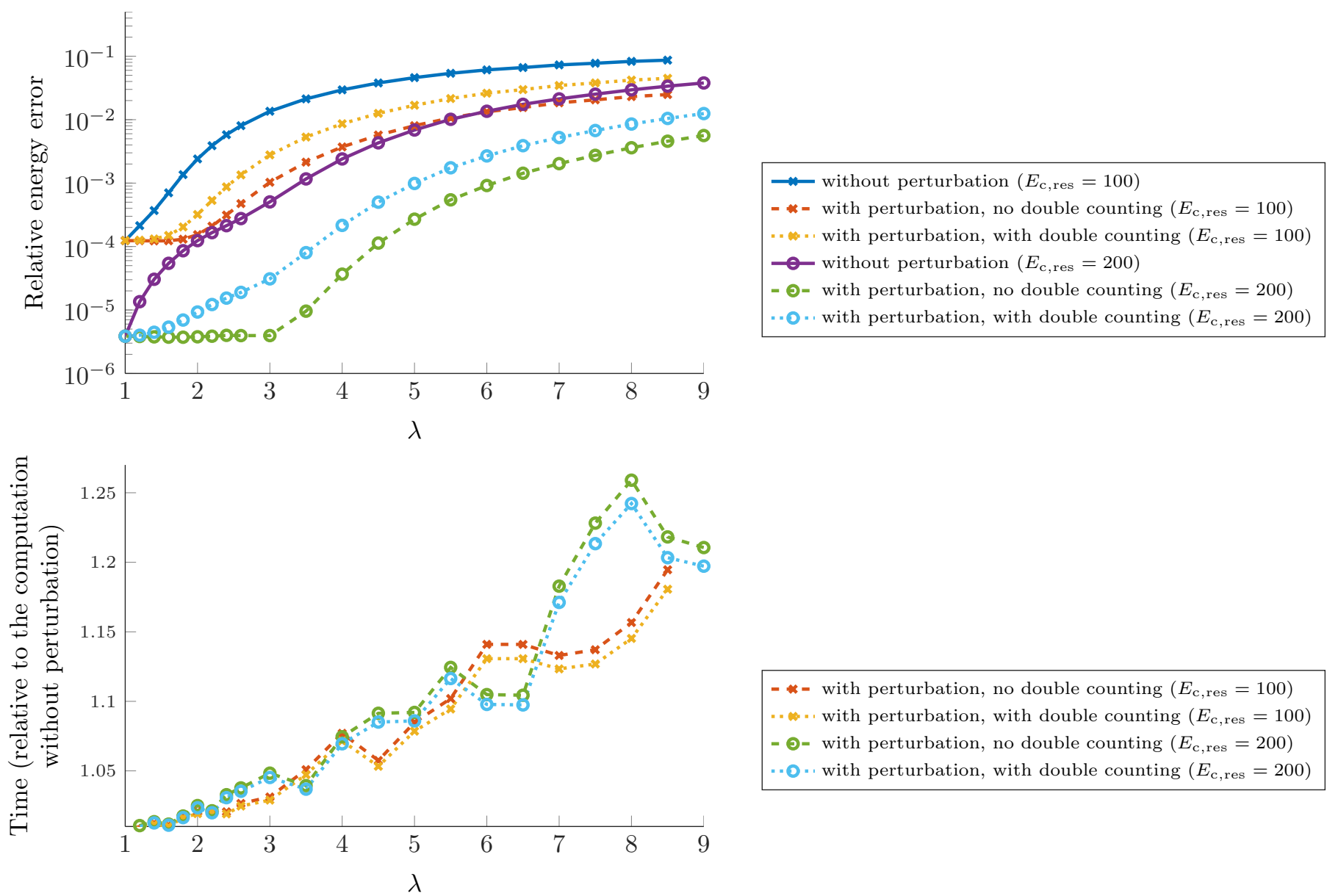

$-*-$ with perturbation, no double counting $\left(E_{\mathrm{c}, \mathrm{res}}=100\right)$
$=-$ with perturbation, with double counting $\left(E_{\mathrm{c}, \mathrm{res}}=100\right)$
$=0-$ with perturbation, no double counting $\left(E_{\mathrm{c}, \mathrm{res}}=200\right)$
$=0$ - with perturbation, with double counting $\left(E_{\mathrm{c}, \mathrm{res}}=200\right)$

Figure 2: Simulations with a constant $E_{\mathrm{c}, \mathrm{res}}$ (alanine molecule).

and is less than $3 \%$ of the total computational time for $\lambda=1.4$ and $\lambda=2$, and no more than $8 \%$ of the total computational time for $\lambda=3$. The computational time is always smaller for the energy formula (23) based on double counting than for the energy formula (22).

For all the values of $E_{\mathrm{c}}$ tested, the best improvement is given either by $\lambda=2$ or $\lambda=1.4$, which drives us into using mostly small values of $\lambda$, as the computations are also less expensive in memory.

Concerning the two possible energy formulas for computing the post-processed energy, the energy formula (22) gives better results than (23). Note that the theoretical results obtained so far are only concerned with formula (22). However the formula (23) based on double counting is a little cheaper to compute to evaluate and also leads to an improvement of the energy.

In conclusion, it seems that whenever the coarse solution is accurate enough, the use of the perturbation method to post-process the solution improves the energy by a factor typically of more than 10. Since the computational cost of this post-process is negligible, it is possible to get the same accuracy as a solution computed on a large grid by first doing a smaller computation on a coarse grid and then post-processing the coarse solution using the perturbation method. This approach is much cheaper than the computation of the solution directly on a grid of size $E_{\mathrm{c}, \text { res }}$.

\section{Acknowledgements}

This work was partially undertaken in the framework of CALSIMLAB, supported by the public grant ANR-11-LABX- 0037-01 overseen by the French National Research Agency (ANR) as part 

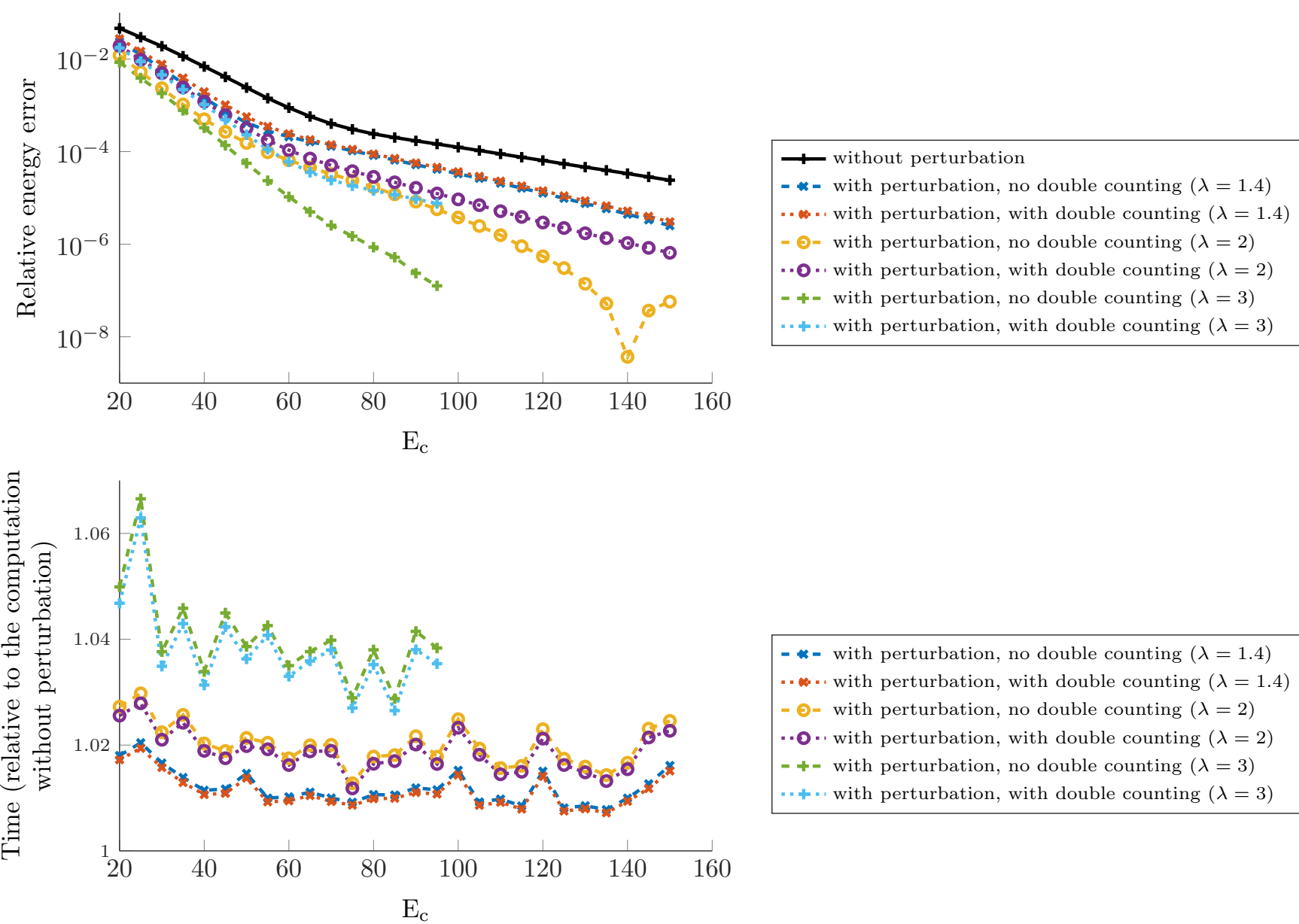

- $*$ - with perturbation, no double counting $(\lambda=1.4)$
- - - with perturbation, with double counting $(\lambda=1.4)$
- 0 - with perturbation, no double counting $(\lambda=2)$
-+- with perturbation, with double counting $(\lambda=2)$
$=+\cdots$ with perturbation, with double counting $(\lambda=3)$

Figure 3: Simulations with a constant $\lambda$ (alanine molecule).

of the Investissements d'avenir program (reference: ANR-11-IDEX-0004-02). Financial support by the ANR grants Manif and Becasim is also acknowledged.

\section{References}

[1] M. Born and J.R. Oppenheimer, Zur Quantentheorie der Molekeln, Ann. Physik 84 (1927) 457-484.

[2] K. Burke, Perspective on density functional theory, The Journal of chemical physics, 136(15), (2012),150901.

[3] E. Cancès, R. Chakir, and Y. Maday, Numerical analysis of the planewave discretization of some orbital-free and Kohn-Sham models, ESAIM: Mathematical Modelling and Numerical Analysis, 46(02), (2012), 341-388.

[4] E. Cancès, M. Defranceschi, W. Kutzelnigg, C. Le Bris and Y. Maday, Computational quantum chemistry: a primer, in Handbook of numerical analysis, Volume X, pp 3-270, North-Holland, Amsterdam, 2003.

[5] E. Cancès, G. Dusson, Y. Maday, B. Stamm and M. Vohralík, On the use of the perturbation method in planewave numerical simulations of linear and non linear Schrödinger equations, in preparation.

[6] E. Cancès and N. Mourad, A mathematical perspective on density functional perturbation theory, Nonlinearity 27 (2014) 19992033.

[7] R.M. Dreizler and E.K.U. Gross, Density functional theory, Springer 1990.

[8] X. Gonze, Perturbation expansion of variational principles at arbitrary order, Phys. Rev. A 52 (1995) 1086;

X. Gonze, Adiabatic density-functional perturbation theory, Phys. Rev. A 52 (1995) 1096.

[9] J. Harris, Simplified method for calculating the energy of weakly interacting fragments, Phys. Rev. B 31(4), (1985) 1770.

[10] P. Hohenberg and W. Kohn, Inhomogeneous electron gas, Phys. Rev. 136 (1964) B864-B871.

[11] W. Kohn and L.J. Sham, Self-consistent equations including exchange and correlation effects, Phys. Rev. 140 (1965) A1133-A1138.

[12] W.A. Lester Jr. (ed.), Recent advances in Quantum Monte Carlo methods, World Sientific 1997.

[13] W.A. Lester Jr., S.M. Rothstein and S. Tanaka (eds.), Recent advances in Quantum Monte Carlo methods, Part II, World Sientific 2002.

[14] M. Levy, Universal variational functionals of electron densities, first order density matrices, and natural spin-orbitals and solution of the V-representability problem, Proc. Natl. Acad. Sci. USA 76 (1979) 6062-6065. 
[15] E.H. Lieb, Density Functional for Coulomb systems, Int. J. Quant. Chem. 24 (1983) 243-277.

[16] J. P. Perdew and A. Zunger, Self-interaction correction to density-functional approximations for many-electron systems, Physical Review B, 23(10), (1981), 5048.

[17] N. Troullier and J.L. Martins, A straightforward method for generating soft transferable pseudopotentials, Solid State Comm. 74 (1990) 613-616.

[18] S. Valone, Consequences of extending 1matrix energy functionals from purestate representable to all ensemble representable 1 matrices, J. Chem. Phys. 73 (1980) 1344-1349.

[19] C. Yang, J. C. Meza, B. Lee, and L.W. Wang, KSSOLV—a MATLAB toolbox for solving the Kohn-Sham equations, ACM Transactions on Mathematical Software (TOMS), 36(2),(2009) 10. 